\title{
A REPRESENTAÇÃO DO CUIDAR NA IMAGEM CULTURAL DA ENFERMAGEM*
}

\author{
Maria Jésia Vieira**
}

VIEIRA, M.J. A representação do cuidar na imagem cultural da enfermagem. Rev.latino-am.enfermagem, Ribeirão

Preto, v. 7, n. 5, p. 25-31, dezembro 1999.

Foram analisados dados de histórias de vida de enfermeiros de Sergipe, sobre a imagem cultural da Enfermagem percebida na escolha da profissão, visando ajudar alunos nesta identificação. A amostra foi composta de quatro grupos cronológicos, desde aposentados até 5 anos de formados, que escolheram a profissão como opção preferencial. Usou-se a abordagem das Representações Sociais nos sistemas culturais, e no contexto sócio histórico. A análise foi feita na modalidade categorial temática e apresentados os dados em forma de gráficos e tabelas. Entre os traços e complexos identificados destacase o "cuidar", com os atributos de conhecimento científico e humanismo, identificado por palavras e expressões que vão de caritativo, religioso, a cientifico, profissionalizado, com permanencias e diversidades no tempo.

UNITERMOS: cuidar, imagem, enfermagem

\section{INTRODUÇÃO}

Alunos iniciantes do curso de Enfermagem têm muitas vezes demonstrado dificuldades em relação à caracterização cultural da profissão escolhida, gerando inseguranças, inclusive por terem feito uma escolha num momento precoce de vida, sem contarem com a orientação de que necessitam. A literatura disponível para ajudar na orientação dos mesmos fala do cuidar como a essência da Enfermagem (HORTA, 1979; VARGENS, 1996), do cuidado ligado a cada cultura (LEININGER, 1978), da história cultural da Enfermagem (ALMEIDA \& ROCHA, 1986), do cuidado sob a perspectiva de gênero (COLLIÈRE, 1989), dos significados do cuidado (REINERS, 1995; WALDOW, 1998), mas não se tem identificado trabalhos que falem de traços e complexos culturais que compõem a imagem da profissão, com permanências e diversidades no tempo.

Entende-se que cada cultura tem traços permanentes e transitórios no tempo e espaço, conforme LARAIA (1994), entre outros, e que o estudo dos mesmos, em relação à profissão, pode ajudar esses alunos a reafirmar ou repensar a escolha feita. As permanências, segundo este autor, evidenciam a especificidade de uma cultura, e as diversidades, seu caráter dinâmico.

Assim, estudou-se numa população de Enfermeiros de Sergipe, que escolheram a Enfermagem como opção preferencial, os traços e complexos percebidos por eles quando da escolha da profissão, e as motivações para esta escolha. O estudo foi feito usandose a metodologia de história de vida, dividindo-se a população em grupos cronológicos, por tempo de exercício profissional, afim de entender as permanências e/ou transitoriedade destes traços de acordo com o tempo.

Entre os traços e complexos permanentes desta população de enfermeiros, formados entre 1952 e 1989 , encontrou-se o "cuidar", com atributos diversos, entre os quais, o humanismo e o conhecimento científico, cuja configuração, diferenciada por grupo cronológico, será discutida especificamente neste trabalho. A análise da representação deste "cuidar" está sendo feita com base nos sistemas culturais estudados por MORAIS (1992), principalmente no sistema adaptativo, coincidindo e incidindo sobre seu mundo; no sistema associativo, na relação com o paciente; e no sistema ideológico, vendo este cuidar como filosofia e como ciência.

\section{METODOLOGIA}

As considerações feitas apontaram como perspectiva teórica e metodológica, para este estudo, as "Representações Sociais" conforme explicitadas por MOSCOVICI (1978; 1988).

\footnotetext{
* dados extraídos da coleta realizada para a tese de doutorado

** Enfermeira, Professor Adjunto do Departamento de Enfermagem e Nutrição do Centro de Ciências Biológicas e da Saúde da Universidade Federal de Sergipe. Doutoranda do Programa Interunidades de Doutoramento em Enfermagem da Escola de Enfermagem de Ribeirão Preto da Universidade de São Paulo
} 
Utilizou-se as entrevistas sobre a história de vida das pessoas incluídas na amostra, na linha da história nova ou história das mentalidades, articulando-a com a história cultural da Enfermagem. Seguiu-se os procedimentos metodológicos recomendados por MEIHY (1996) na coleta de dados, processamento, validação e autorização de uso pelos colaboradores.

A amostra foi intencional, a partir da pessoa identificada como sendo a mais antiga enfermeira, entre as residentes no Estado de Sergipe, e a partir daí, compondo-se a rede de informantes pelas indicações que cada colaborador fazia de outros possíveis. Mediante concordância destes, fazia-se inicialmente uma entrevista semi estruturada para identificar as pessoas que escolheram a Enfermagem como opção preferencial, e a seguir a entrevista com história de vida para identificar a imagem cultural da profissão percebida pelo colaborador no momento de escolha da mesma. Colocando as questões em bloco, conforme recomenda MEIHY (1996), pediase a cada colaborador que falasse sobre sua própria visão relacionada à imagem cultural da profissão, na época da sua escolha, e sobre possíveis fatos, circunstâncias, eventos, pessoas, vultos, características relacionadas com a imagem cultural da profissão que tivessem tido influência marcante na sua escolha pessoal.

As entrevistas foram gravadas, digitadas, conferidas pelos respectivos entrevistados tendo cada um autorizado por escrito o seu uso sem restrições.

Em se tratando de um estudo da imagem cultural, trabalhou-se com quatro extratos de população agrupados por tempo cronológico de exercício profissional: aposentados, exercentes da profissão há mais de 25 anos, exercentes entre 15 a 25 anos, e exercentes entre 5 a menos de 15 anos, incluindo-se em cada grupo pelo menos uma pessoa da área assistencial hospitalar, uma da área de saúde pública, uma da área de ensino e uma da área de coordenação ou administração central de instituições de saúde.

A coleta de dados foi realizada na cidade de Aracaju, capital do Estado de Sergipe, no período de junho a setembro de 1997. Foram entrevistadas 52 pessoas, das quais somente 19 preencheram o critério da opção preferencial, sendo 18 mulheres e 01 homem. Esta coleta foi encerrada no momento em que foram preenchidos todos os grupos da amostra com seus respectivos componentes.

A análise das entrevistas foi feita seguindo a modalidade de análise categorial temática proposta por BARDIN (s/d) e adaptada para este estudo, constando de pré-análise, exploração do material e tratamento dos resultados agrupados em categorias, para a seguir fazer a montagem dos temas, dentre os quais elegeu-se o "cuidar" para ser discutido neste trabalho. As categorias foram submetidas a análise de freqüência, sendo apresentadas em gráficos e tabelas contendo valores em números absolutos e percentual.

\section{ANÁLISE DOS DADOS}

Os dados, a seguir, indicam os traços e complexos culturais ligados ao "cuidar", percebidos e referidos pelos entrevistados durante o relato de sua história de vida enfocando a escolha da profissão. Com base em SPINK (1994), pode-se afirmar que estes traços e complexos, embora referidos no tempo presente, onde está-se dando a interação, expressam as representações sociais destes colaboradores, ancoradas no tempo vivido, ou do seu processo de socialização, e no tempo longo, com suas influências históricas e sócio culturais.

\section{Quadro 1 - Freqüência percentual dos traços e complexos culturais percebidos e relatados pelos enfermeiros dos quatro grupos cronológicos no momento da escolha da profissão}

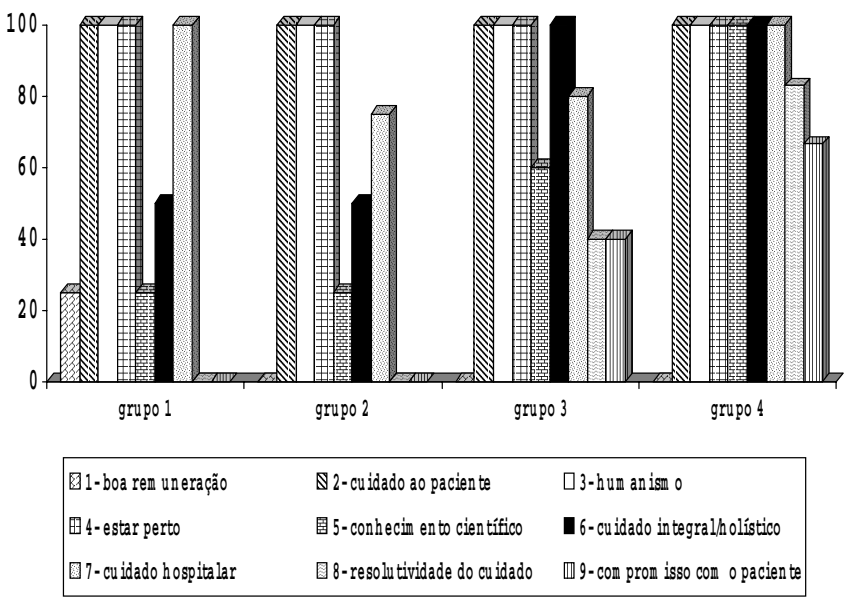

Legenda: grupo $1(\mathrm{~N}=4)$ aposentados. Graduados entre 1952 e 1972 (45 a 25 anos de graduados)

grupo $2(\mathrm{~N}=4)$ exercentes há mais de 25 anos. Graduados entre 1961 a 1971

grupo $3(\mathrm{~N}=5)$ exercentes entre 15 a 25 anos. Graduados entre 1974 a 1981

grupo $4(\mathrm{~N}=6)$ exercentes entre 5 e menos de 15 anos. Graduados entre 1983 a 1989

Conforme pode ser observado no Quadro 1, a imagem do cuidado ao paciente e seu atributo de humanismo foram referidos por todos os entrevistados, em todos os grupos, sendo pois traços culturais permanentes, inclusive na literatura consultada. De igual forma, o estar perto evidenciou-se como um traço permanente na amostra, já que todos os entrevistados referem o "cuidar estando perto" como característica que diferencia a Enfermagem das outras profissões de cuidado. Esta referência também foi encontrada na população estudada por VARGENS (1996). 
Este cuidar tem uma forte conotação de cuidado ao paciente no hospital, também ancorado no contexto histórico social da profissão, e se caracteriza como cuidado integral ou holístico, numa rejeição crescente ao cuidado dividido por tarefas, sendo o primeiro termo referido mais pelos grupos 1,2 , e 3 , e o cuidado holístico mais referido pelo grupo 4, evidenciando reflexos das discussões filosóficas sobre o cuidado, relacionadas ao tempo de escolha profissional de cada um destes grupos.

As referências à resolutividade do cuidado e ao compromisso com o paciente só apareceram nos grupos 3 e 4, o que não significa que na prática estes atributos não existam nos outros grupos, mas tão somente que sua representação social não se manifesta com intensidade no discurso anterior aos anos 70 .

Os atributos de humanismo e conhecimento científico como componentes do cuidar em enfermagem têm sido objeto de análise na categoria profissional, vez que o segundo, durante muito tempo, não teve a necessária evidência, prejudicando, inclusive, a imagem da profissão no conjunto das profissões de saúde. Esta discussão, acompanhada de uma mudança crescente na configuração da imagem cultural da profissão, e conseqüentemente na representação social dos componentes da amostra, no momento da escolha, fica evidenciada no Quadro 2, onde pode-se perceber uma permanência temporal do atributo humanismo e uma curva crescente das referências ao conhecimento científico como atributo do cuidado, desde o grupo 1 até o grupo 4 .
Quadro 2 - Freqüência percentual dos atributos humanismo e conhecimento científico como componentes do cuidar em enfermagem segundo percepção dos quatro grupos cronológicos no momento da escolha da profissão

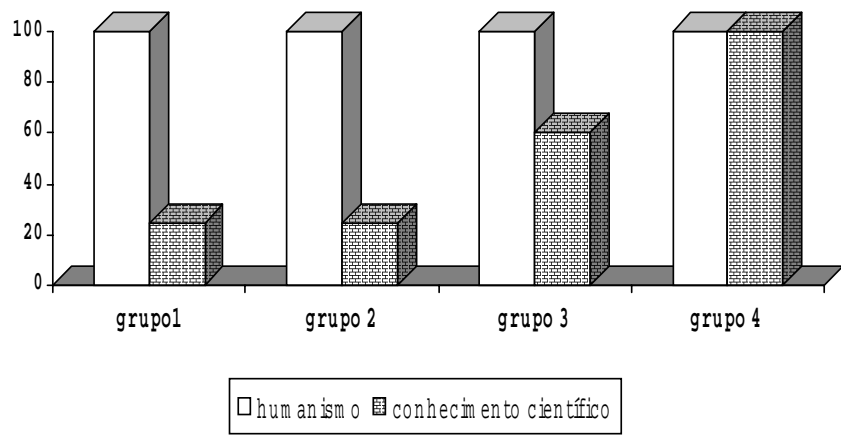

Legenda: grupo $1(\mathrm{~N}=4)$ aposentados. Graduados entre 1952 e 1972 (45 a 25 anos de graduados)

grupo $2(\mathrm{~N}=4)$ exercentes há mais de 25 anos. Graduados entre 1961 a 1971

grupo $3(\mathrm{~N}=5)$ exercentes entre 15 a 25 anos. Graduados entre 1974 a 1981

grupo $4(\mathrm{~N}=6)$ exercentes entre 5 e menos de 15 anos. Graduados entre 1983 a 1989

Esses atributos, humanismo e conhecimento científico, foram referidos com palavras e expressões diferenciadas, refletindo a ideologia predominante do contexto sócio histórico no qual estas representações se ancoram, indo desde o sentido caritativo, religioso, ao científico, holístico, profissionalizado, como pode ser verificado nas Tabelas 1 e 2 .

Tabela 1 - Palavras e expressões utilizadas pelos entrevistados dos quatro grupos cronológicos para se referirem ao "conhecimento científico" como componente do cuidar em enfermagem

\begin{tabular}{l|c|c|c|c|c|c|c|c|c|c}
\hline \multirow{2}{*}{ Palavras/exp. } & \multicolumn{2}{|c|}{ Grupos cronológ. } & \multicolumn{2}{c|}{ Grupo 2 } & \multicolumn{2}{c|}{ Grupo 3 } & \multicolumn{2}{c|}{ Grupo 4 } & \multicolumn{2}{c}{ Total } \\
\cline { 2 - 10 } & $\mathrm{N}^{\circ}$ & $\%$ & $\mathrm{~N}^{\circ}$ & $\%$ & $\mathrm{~N}^{\circ}$ & $\%$ & $\mathrm{~N}^{\circ}$ & $\%$ & $\mathrm{~N}^{\circ}$ & $\%$ \\
\hline Conhecimento científico & 01 & 25,0 & 01 & 25,0 & 03 & 60,0 & 06 & 100,0 & 11 & 57,9 \\
\hline - competência & 01 & 25,0 & & & 02 & 40,0 & 04 & 66,7 & 07 & 36,8 \\
\hline - estudos/preparo & 01 & 25,0 & & & & & 02 & 33,3 & 03 & 15,8 \\
\hline - ciênc. Do cuidado + humanas & 01 & 25,0 & 01 & 25,0 & 02 & 40,0 & 02 & 33,3 & 06 & 31,6 \\
\hline - conh. Simples ao complexo & & & 01 & 25,0 & & & 03 & 50,0 & 04 & 21,0 \\
\hline - conhecimento abrangente & & & & & & & 03 & 50,0 & & \\
\hline Total de entrevistados & 04 & & 04 & & 05 & & 06 & & 19 & \\
\hline
\end{tabular}

Legenda: grupo $1(\mathrm{~N}=4)$ aposentados. Graduados entre 1952 e 1972 (45 a 25 anos de graduados) grupo $2(\mathrm{~N}=4)$ exercentes há mais de 25 anos. Graduados entre 1961 a 1971 grupo $3(\mathrm{~N}=5)$ exercentes entre 15 a 25 anos. Graduados entre 1974 a 1981 grupo $4(\mathrm{~N}=6)$ exercentes entre 5 e menos de 15 anos. Graduados entre 1983 a 1989 
Conforme já expresso no Quadro 2, o conhecimento científico, como atributo do cuidar em Enfermagem, é predominante no grupo 4. Os colaboradores que fizeram referência ao mesmo falam da necessidade do seu crescimento contínuo na prática diária para que se possa ter segurança nas ações desenvolvidas, poder de argumentação, desenvolvimento do cuidado holístico, implementação de ações sinérgicas em benefício do paciente, conseguir resolutividade no cuidado, ter o respeito dos pacientes e dos outros profissionais, e para que se possa desenvolver a pesquisa na Enfermagem.

Tabela 2 - Palavras e expressões utilizadas pelos entrevistados dos quatro grupos cronológicos para se referirem ao "humanismo" como componente do cuidar em enfermagem

\begin{tabular}{|c|c|c|c|c|c|c|c|c|c|c|}
\hline \multirow{2}{*}{ Palavras/exp. } & \multicolumn{2}{|c|}{ Grupo 1} & \multicolumn{2}{|c|}{ Grupo 2} & \multicolumn{2}{|c|}{ Grupo 3} & \multicolumn{2}{|c|}{ Grupo 4} & \multicolumn{2}{|c|}{ Total } \\
\hline & $\mathrm{N}^{\circ}$ & $\%$ & $\mathrm{~N}^{\circ}$ & $\%$ & $\mathrm{~N}^{\circ}$ & $\%$ & $\mathrm{~N}^{\circ}$ & $\%$ & $\mathrm{~N}^{\circ}$ & $\%$ \\
\hline Humanismo & 04 & 100,0 & 04 & 100,0 & 05 & 100,0 & 06 & 100,0 & 19 & 100,0 \\
\hline Doação & 03 & 75,0 & & & & & & & 03 & 15,8 \\
\hline Caridade & 02 & 50,0 & & & & & & & 02 & 10,5 \\
\hline Religião & 02 & 50,0 & & & & & & & 02 & 10,5 \\
\hline Missão & 01 & 25,0 & 01 & 25,0 & & & & & 02 & 10,5 \\
\hline Servir ao paciente & 02 & 50,0 & 03 & 75,0 & 01 & 20,0 & & & 06 & 31,6 \\
\hline Proteção & 01 & 25,0 & 01 & 25,0 & 01 & 20,0 & & & 03 & 15,8 \\
\hline Transmitir calor humano & 02 & 50,0 & 02 & 50,0 & 04 & 80,0 & 05 & 83,3 & 13 & 68,4 \\
\hline Ser humano como foco & 03 & 75,0 & 01 & 25,0 & 01 & 20,0 & 05 & 83,3 & 10 & 52,6 \\
\hline Ajudar & 02 & 50,0 & & & 05 & 100,0 & 04 & 66,6 & 11 & 57,9 \\
\hline Dedicação ao paciente & 04 & 100,0 & 04 & 100,0 & 03 & 60,0 & 04 & 66,6 & 15 & 78,9 \\
\hline Disponibilidade & & & & & 01 & 20,0 & 02 & 33,3 & 03 & 15,8 \\
\hline Cuidar & 04 & 100,0 & 04 & 100,0 & 05 & 100,0 & 06 & 100,0 & 19 & 100,0 \\
\hline Cuidado integral/holístico & 02 & 50,0 & 02 & 50,0 & 04 & 80,0 & 06 & 100,0 & 14 & 73,7 \\
\hline Cuid. bio-psico-emocional & & & & & 01 & 20,0 & 04 & 66,6 & 05 & 26,3 \\
\hline Total de entrevistados & 04 & & 04 & & 05 & & 06 & & 19 & \\
\hline
\end{tabular}

Legenda: grupo $1(\mathrm{~N}=4)$ aposentados. Graduados entre 1952 e 1972 (45 a 25 anos de graduados)

grupo $2(\mathrm{~N}=4)$ exercentes há mais de 25 anos. Graduados entre 1961 a 1971

grupo $3(\mathrm{~N}=5)$ exercentes entre 15 a 25 anos. Graduados entre 1974 a 1981

grupo $4(\mathrm{~N}=6)$ exercentes entre 5 e menos de 15 anos. Graduados entre 1983 a 1989

Nesta tabela foram conservadas literalmente as palavras e expressões dos entrevistados vez que as mesmas refletem o sentido ideológico ligado à orientação filosófica predominante em cada época, embora, na prática, algumas delas tenham a mesma objetivação, tais como dedicação e disponibilidade. Outras trazem forte conotação da ideologia religiosa caritativa, tais como: doação, caridade, religião, missão, servir; e outras são englobadas no cuidar, no cuidado integral e/ou holístico, ou no cuidado bio-psico-emocional. As mais ligadas à conotação religiosa caritativa são predominantes no primeiro grupo, decrescendo a partir do segundo. As palavras humanismo e cuidar aparecem em $100 \%$ da amostra. 
Os dados apresentados evidenciaram o "cuidar" como um traço permanente na caracterização da profissão. Este "cuidar" é defendido por HORTA (1979), como sendo a essência da profissão, do qual derivam todas as funções, tendo seu enfoque no ser humano, e não na sua doença.

Os atributos de humanismo, e o estar perto, relacionados ao cuidar, também se mostraram permanentes na imagem cultural da profissão, diferente do atributo conhecimento científico, que ganha mais expressividade a partir do grupo 3 , cujo período de escolha da profissão, anos 70 a 80 , coincide com as referências da literatura sobre o aumento da produção científica dos enfermeiros no país, conforme ALMEIDA \& ROCHA (1986), entre outros. É neste período que se pode perceber, na literatura, e bem assim nos temas oficiais dos Congressos Brasileiros de Enfermagem, os esforços e discussões da categoria no sentido de tornar cada vez mais evidente e efetiva a característica de cientificidade da profissão.

Foi também neste período, conforme os mesmos autores, que se implementou a pós-graduação, foram elaboradas e divulgadas teorias de âmbito nacional e internacional, constituindo-se o que estes denominam de "o corpo de conhecimentos específicos da enfermagem" (ALMEIDA \& ROCHA, 1986, p.87).

O humanismo, entretanto, sempre esteve no imaginário sócio-cultural, a partir do cuidado prestado pelas mães, pelos componentes de instituições caritativo religiosas, dando inicialmente à profissão uma aproximação cultural com esse cuidado não profissionalizado. A este respeito ALCÂNTARA (1963) fez referências identificando o cuidado caritativo como valorizado pela sociedade, e o cuidado profissionalizado como desprestigiado, já que culturalmente ligado às conotações atribuídas ao trabalho da mulher.

O humanismo tem, pois, forte argumentação valorativa neste imaginário, e é interessante perceber que o movimento crescente de cientificidade não diminui esta força, mas realiza um movimento de conjunção que ganha maior expressividade com a expansão e divulgação do paradigma holístico nas ciências, paradigma este que se caracteriza pelo aspecto de complementaridade, pelo diálogo entre as diversas formas de conhecimento, e pela visão do homem em suas múltiplas dimensões (CREMA, 1991).

Assim, as palavras e expressões utilizadas em referência ao conhecimento científico trazem a conotação desta complementaridade, tais como "conhecimento das ciências do cuidado e das ciências humanas", "conhecimento abrangente", "conhecimento do simples ao mais complexo", reforçados pela expressão "competência no exercício da profissão".

Também entre as palavras e expressões utilizadas pelos colaboradores para se referirem ao humanismo como atributo do cuidar pode-se perceber esta visão do ser humano integral, mas, conforme sua ancoragem histórico social e os movimentos desenvolvidos pela profissão, através dos tempos, a objetivação se manifesta conforme tabela 1, como caridade, maternalismo, relação de ajuda e cuidado holístico, tendo estas conotações, também uma configuração temporal nos grupos onde são identificadas.

O cuidado hospitalar, além de sua ancoragem histórica nas instituições de abrigo, reclusão e proteção social a que se refere FOUCAULT (1993), tem, também, no Brasil, seu crescimento sob influência do modelo assistencial de saúde, implementado pelo Ministério de Previdência e Assistência Social, que privilegiou a assistência curativa de caráter individual, dirigida, principalmente, à recuperação da força de trabalho, conforme FERNANDES (1985).

A resolutividade do cuidado e o compromisso social, evoluindo de forma crescente do terceiro ao quarto grupo, guardam relação com os movimentos sociais, deflagrados em várias vertentes no país. Entre estas, a discussão da saúde como um direito e a assistência como um dever, ao invés de uma concessão, preconizados pela Conferência de Alma-Ata (OMS/UNICEF, 1978), e levadas a efeito não só pelas categorias profissionais de saúde, mas por toda a sociedade, e que teria mais tarde sua maior expressividade, no país, na $8^{\text {a }}$ Conferência Nacional de Saúde em 1986. No âmbito das categorias profissionais, entretanto, esta discussão já fazia eco, e na Enfermagem, especificamente em 1979 era tema do Congresso Brasileiro de Enfermagem realizado em Fortaleza (ASSOCIAÇÃO BRASILEIRA DE ENFERMAGEM - ABEn, 1982).

Poder-se-ia, entretanto questionar se o jovem vestibulando teria acesso a estas informações. Novamente a teoria da Representação Social pode explicar a percepção e o processamento de informações na intercecção do individual com o social, pois não só o jovem, como todos os seres sociais percebem, ancoram e objetivam, realizando a ação de aproximação ou rejeição dos elementos conforme a significação que lhes atribui (VALA, 1993).

Entende-se, pois, que o jovem percebia as atitudes expressas pelos profissionais, conforme sua orientação filosófica, principalmente porque, neste trabalho, foram incluídos apenas aqueles que tinham a enfermagem como opção preferencial, o que pressupõe um conhecimento, mesmo que vago, e/ou uma aproximação de pessoas ou traços da profissão. Um exemplo disso pode ser visto citando um dos 
colaboradores, quando fala das enfermeiras, através das quais tomou conhecimento da profissão: “... elas falavam dos pacientes que tinham se recuperado, ... dos cuidados... era um cuidar de forma efetiva".

Assim, as permanências e mudanças culturais identificadas neste trabalho, evidenciam a dinamicidade da cultura, nos seus sistemas adaptativo, associativo e ideológico, a que se refere MORAIS (1992), e consoante as afirmações de LEININGER (1978), de que cada cultura tem maneiras próprias de definir, compreender, expressar e explicar a saúde e a doença, sendo assim, o cuidado, um fenômeno culturalmente construído.

Neste sentido, as representações sociais dos indivíduos sobre a profissão escolhida abrangeram: o campo do conhecimento, através das informações veiculadas pela sociedade em cada época; o campo do valor, com suas palavras ligadas às conotações ideológicas que as ancoram, e o campo da ação de aproximação do objeto representado, campos, estes, referidos por LAPLANTINE (1994). Estas representações situam-se dentro do processo de socialização, onde, segundo BERGER \& LUCKMANN (1973), cada indivíduo se coloca com suas capacidades, tendências, motivações e possibilidades, para exercer um papel específico dentro do seu grupo social, exteriorizando-se nele e interiorizando seus valores.

\section{CONSIDERAÇÕES FINAIS}

Conforme foi possível perceber, através das tabelas e quadros apresentados, o contexto sócio histórico e a trajetória da cultura de cada época imprimiram características próprias ou modificaram algumas formas de objetivação da imagem da profissão nos quatro grupos estudados. Pode-se perceber, entretanto, como principal núcleo de sentido o "cuidar", qualificado com alguns atributos como o humanismo e o estar perto, como traço permanente da imagem cultural, o conhecimento científico como traço transitório crescente, e a perspectiva permanente da visão do homem como um ser integrado.

O trabalho como um todo também identificou outras categorias de análise que foram reunidas em temas tais como a identificação, a cultura e a transcendência, que serão objeto de trabalhos posteriores.

\section{AGRADECIMENTOS}

A autora agradece ao Prof. Dr. Lewis Joel Greene, e ao grupo da disciplina RGO 826 - Redação de Documentos Científicos, pelas discussões constantes durante a elaboração deste.

Agradece, também à Prof. Dra. Maria Cecília Manzolli, orientadora da tese, de cuja coleta de dados este trabalho foi extraído.

\section{THE REPRESENTATION OF CARE IN NURSING CULTURAL IMAGE}

We analyzed Sergipan nurses' life stories, related to the cultural image of Nursing perceived in their professional choice, in order to assist students in this professional identification. Subjects were divided into four chronological groups, from retired nurses to those who had been graduated for 5 years, all of whom chose Nursing as their preferred career. We used the Social Representation approach in the cultural systems to analyze the life stories. Findings were analyzed through categories and themes, and it was presented by graphics and tables. Care was identified as a permanent cultural trace and it was verbalized using words from charity, religion to scientific Nursing and professionalism, to describe scientific knowledge and humanism, with temporal configuration.

KEY WORDS: care, image, nursing

\section{LA REPRESENTACIÓN DEL CUIDAR EN LA IMAGEN CULTURAL DE LA ENFERMERÍA}

Fueran analizados datos de historias de vida de enfermeros sergipanos, que tuvieron la Enfermería como opción profesional de preferencia, con el propósito de ayudar alumnos en esta identificación profesional. Fueran entrevistados cuatro grupos desde los jubilados hasta los que tenían 5 años de graduados. Se emplea el abordaje de las Representaciones Sociales, y de los sistemas culturales en su contexto socio histórico. Fueron analizados las categorías y temas de los relatos, empleando gráficos y tablas para su presentación. El cuidar se identificó entre los trazos culturales permanentes. Se identificaron palabras y expresiones que significan desde caridad, hasta Enfermería científica y profesionalismo. 


\section{REFERÊNCIAS BIBLIOGRÁFICAS}

01. ALCÂNTARA, G.de. Enfermagem moderna como categoria profissional: obstáculos à sua expansão na sociedade brasileira. Ribeirão Preto, 1963. 125p. Tese (cátedra)- Escola de Enfermagem de Ribeirão Preto, Universidade de São Paulo.

02. ALMEIDA, M.C.P.de; ROCHA, J.S.Y. O saber da enfermagem e sua dimensão prática. São Paulo: Cortez, 1986. 128p.

03. ASSOCIAÇÃO BRASILEIRA DE ENFERMAGEM - ABEn. 33 anos de Congresso Brasileiro de Enfermagem: retrospectiva. Porto Alegre: Pallottti, 1982. 205p.

04. BARDIN, L. Análise de conteúdo. Lisboa: Ed.70, s/ d. 229p. Traduzido de: L'Analyse de Contenu.

05. BERGER, P.L.; LUCKMANN, T. A construção social da realidade. 11. ed. Petrópolis: Vozes, 1994. 247p. Traduzido de: The Social Construction of Reality, 1966.

06. COLLIÈRE, M.F. Promover a vida: da prática das mulheres de virtude aos cuidados de Enfermagem. Lisboa: Sindicato dos Enfermeiros portugueses, $1989.385 \mathrm{p}$.

07. CREMA, R. Abordagem holística: integração do método analítico e sintético. In: BRANDÃO, D.M.S.; CREMA, R. O novo paradigma holístico. 2. ed. São Paulo: Summus, 1991. p. 8399.

08. FERNANDES, J.D. A enfermagem no ontem, no hoje e no amanhã. Rev.Bras.Enfermagem, Brasília, v. 38, n. 1, p. 43-48, jan./mar. 1985.

09. FOUCAULT, M. Microfísica do poder. 11. ed. Rio de Janeiro: Graal, 1993. p. 79-112.

10. HORTA, W.de A. Processo de Enfermagem. São Paulo: EPU/EDUSP, 1979. p. 3-41.

11.LAPLANTINE, F. Antropologie des systèmes de représentations de la maladie. In: JODELET, D.(org.). Les representations socialles. 4.ed. Paris: Presses Universitaires de France, 1994. p. 277-298.
12. LARAIA, R.de B. Cultura: um conceito antropológico. 9.ed. Rio de Janeiro: Jorge Zahar, 1994. 116p.

13. LEININGER, M. Transcultural Nursing: concepts, theories and practice. New York: John Wiley, 1978. p. 7-120.

14. MEIHY, J.C.S.B. Manual de história oral. São Paulo: Loyola, 1996. 78p.

15. MORAIS, R. de. Estudos de filosofia da cultura. São Paulo: Loyola, 1992. p. 35-70.

16. MOSCOVICI, S. A representação social da Psicanálise. Rio de Janeiro: Zahar, 1978. 291p. Traduzido de: La Psychanalyse - son image et son public.

17. Notes towards a description of Social Representations. Eur.J.Soc.Psychol., v. 18, p. 211-250, 1988.

18. OMS/UNICEF. Conferência Internacional sobre Atenção Primária de Saúde. Alma-Ata: URSS, 1978.

19. REINERS, A.A.O. Cuidado: seu significado para o enfermeiro. Ribeirão Preto, 1995. Dissertação (mestrado)- Escola de Enfermagem de Ribeirão Preto, Universidade de São Paulo. 95p.

20. SPINK, M.J.P. Permanências e diversidades nas representações sociais da hipertensão arterial essencial. Temas Psicologia, São Paulo, n. 2, p. 199-212, 1994.

21. VALA, J. Representações sociais: para uma psicologia social do pensamento social. In: VALA, J.; MONTEIRO, M.B. Psicologia social. Lisboa: Fund.Calauste/Gulbenkein, 1993. p. 353-384.

22. VARGENS, O.M.C. Tentando descobrir um modo de fazer enfermagem sem ser enfermeiro: os conflitos do estudante na construção da imagem da profissão. Ribeirão Preto, 1996. 176p. Tese (doutorado) - Escola de Enfermagem de Ribeirão Preto, Universidade de São Paulo.

23. WALDOW, V.R. Cuidado humano: o resgate necessário. Porto Alegre: Sagra Luzzatto, 1998. p. 93-159. 